\title{
A organização do acesso aos serviços de saúde bucal na estratégia de saúde da família de um município da Bahia
}

The organization of the access to dental health services within the family healthcare strategy in a municipality in Bahia

Roseli Pereira Tavares' ${ }^{1}$ Giovanni Caponi Costa², Michelle Lopes Miranda Falcão³, Patrícia Suguri Cristino ${ }^{4}$

'Graduada em Odontologia pela União Metropolitana de Ensino e Cultura (UNIME) - Lauro de Freitas (BA), Brasil. tavares.roseli@uol.com.br

${ }^{2}$ Graduada em Odontologia pela União Metropolitana de Ensino e Cultura (UNIME) - Lauro de Freitas (BA), Brasil. giovannicaponi@hotmail.com

${ }^{3}$ Mestre em Saúde Coletiva pela Universidade Estadual de Feira de Santana (UEFS) - Feira de Santana (BA), Brasil. Professora do Curso de Odontologia da

Universidade Estadual de Feira de Santana

- Feira de Santana (BA), Brasil.

michellefalcao@gmail.com

${ }^{4}$ Mestre em Odontologia Restauradora pela Universidade de São Paulo (USP)

- Ribeirão Preto (SP), Brasil. Professora da Faculdade de Odontologia da Universidade Federal da Bahia (UFBA) Salvador (BA), Brasil.

patriciasgcristino@gmail.com
RESUMO: O objetivo desse estudo foi conhecer a forma de organização da oferta dos serviços de saúde bucal na Estratégia de Saúde da Família de um município da Bahia. Trata-se de pesquisa qualitativa baseada em entrevistas semiestruturadas com usuários, agentes comunitários de saúde e cirurgiões-dentistas. Os resultados permitiram inferir que a oferta da primeira consulta odontológica aproxima-se mais do modelo tradicional de atendimento à demanda espontânea. Constatou-se que a participação da comunidade na organização dos serviços de saúde bucal é ausente. As barreiras de acesso recaíram sobre a falta de materiais e deficiência na manutenção de equipamentos, além da baixa cobertura do serviço.

PALAVRAS-CHAVE: Agendamento de consultas; Serviços de saúde bucal; Equidade no acesso.

\begin{abstract}
The objective of this study was to investigate the organization of available dental health services within the Family Healthcare Strategy in a Bahia State's municipality. This is a qualitative research study using semi-structured interviews with users, community health agents and dentists. The results showed that the availability of the initial dental consultation is similar to the traditional model of healthcare by spontaneous demand. Community participation in the organization of oral healthcare services proved to be non-existent. Barriers to access are due to the lack of supplies and inadequate equipment maintenance, in addition to the limited services provided.
\end{abstract}

KEYWORDS: Appointments and schedules; Dental Health Services; Equity in Access. 


\section{Introdução}

No ano de 2004, foi proposta a Política Nacional de Saúde Bucal (PNSB), calcada nos princípios doutrinários e organizativos do Sistema Único de Saúde (SUS) e estruturada com o objetivo de ampliar e garantir a atenção odontológica à população, conhecida como Programa Brasil Sorridente (FRAZÃO; NARVAI, 2009).

Após sete anos do início de sua implantação, a PNSB alcançou números importantes no território nacional. Em termos de cobertura dos serviços de saúde bucal na Estratégia de Saúde da Família (ESF), a população coberta passou de $15,2 \%$, em 2004, para 34,7\% em 2011. O número de Centros de Especialidades Odontológicas (CEOs) aumentou de 100 para 853 nesse mesmo período (BRASIL, 2012).

Quanto ao impacto da PNSB sobre as desigualdades em saúde bucal, o incremento de cirurgiôes-dentistas no SUS foi consideravelmente maior nas regióes Norte e Nordeste, e outros estudos corroboram o maior aporte de recursos nas cidades com piores indicadores socioeconômicos, numa tendência pró-equidade (ANTUNES; NARVAI, 2010).

Apesar dos inegáveis avanços da PNSB, pode-se observar que a cobertura dos serviços na atenção básica ainda é baixa no contexto nacional, um fato considerável diante das necessidades acumuladas historicamente pela população brasileira. Diante disso, emerge imediatamente um questionamento: as açóes e serviços da PNSB têm alcançado as pessoas que mais precisam? Se a Atenção Básica deve ser a 'porta de entrada' do sistema de saúde, eis um grande desafio para as Equipes de Saúde Bucal (ESBs), que precisam organizá-la pautando-se também pela equidade.

Num país classificado pelo Relatório de Desenvolvimento Humano da ONU como sendo o $3^{\circ}$ país de maior desigualdade social do mundo no ano de 2010, promover o acesso a quem mais precisa talvez seja o maior imperativo ético e humano de qualquer política pública neste País (PNUD, 2010).

O objetivo deste estudo foi conhecer como as ESBs de um município do estado da Bahia estavam organizando a oferta dos serviços de saúde bucal na ESF no ano de 2011.

\section{Metodologia}

Trata-se de uma pesquisa qualitativa realizada por meio de entrevistas semiestruturadas aplicadas a alguns integrantes das Equipes de Saúde da Família de um Município do estado da Bahia com aproximadamente 163.449 habitantes (IBGE, 2012), cujo serviço de saúde dispóe de nove Equipes de Saúde Bucal (ESBs) Modalidade I - Cirurgião-dentista e Auxiliar de Saúde Bucal - inseridas na ESF e cinco Cirurgióes-dentistas concursados atuando em ambulatório de atendimento a urgências. A cobertura populacional de saúde bucal na ESF no ano de 2011 era de 19\% (BRASIL, 2012).

Diante desse cenário, o critério adotado para seleção dos sujeitos da pesquisa foi possuir experiência relacionada ao objeto de estudo no contexto do serviço de saúde, incluindo os diferentes olhares (MINAYO, 2008) propiciados pelas posiçóes de cada entrevistado na Estratégia de Saúde da Família. Assim, participaram do trabalho três Cirurgióes-dentistas (CDs), três Agentes Comunitários de Saúde (ACS) e seis usuários provenientes de três Unidades de Saúde da Família (USFs) do referido município. Ressalta-se que o tamanho da amostra considerou o critério de saturação preconizado por Minayo (2008).

Este estudo foi aprovado pelo Comitê de Ética em Pesquisa do Instituto Mantenedor de Ensino Superior da Bahia (CEP/IMES) da Faculdade de Tecnologia e Ciência, e autorizado pela Coordenação de Saúde Bucal da Secretaria Municipal de Saúde, a qual elencou as USFs possíveis para o estudo, já que o Município atravessava um momento de transição de substituição de CDs contratados por concursados.

Após esses procedimentos, realizaram-se as entrevistas - áudio-gravadas e posteriormente transcritas - com os doze sujeitos da pesquisa em suas respectivas USFs. O material resultante foi submetido à técnica de análise de conteúdo preconizada por Bardin (1979), tendo nas Diretrizes da Política Nacional de Saúde Bucal (BRASIL, 2013) o horizonte teórico para interpretação.

A partir da leitura do material transcrito, foram construídas três categorias de análise: (1) marcação da primeira consulta odontológica; (2) participação da 
comunidade usuária na organização dos serviços de saúde bucal; e (3) acessibilidade ao serviço de saúde bucal.

As respostas foram selecionadas e transcritas na discussão, preservando-se a identificação de todos os sujeitos participantes, caracterizados por letras e números, a fim de contemplar as diferenciaçóes necessárias. Convencionou-se, então, identificar pela sigla $\mathrm{CD}$ os depoimentos advindos de Cirurgióes-dentistas, pela sigla ACS aqueles procedentes de Agentes Comunitários de Saúde e pela sigla US aqueles que partiram dos Usuários.

\section{Resultados e discussão}

\section{A MARCAÇÃO DA PRIMEIRA CONSULTA ODONTOLÓGICA}

Quando proposto pelo Ministério da Saúde, o indicador de cobertura da primeira consulta odontológica programática deveria representar o percentual de pessoas que receberam uma primeira consulta odontológica realizada com finalidade de diagnóstico e, necessariamente, com elaboração de um plano preventivo-terapêutico (PPT) para atender às necessidades detectadas (BRASIL, 2006).

Quando os usuários e agentes comunitários de saúde foram questionados sobre como são organizadas, distribuídas, as vagas para tratamento odontológico - oferta da primeira consulta odontológica -, encontrou-se:

US1, US2, US5, US6 - todos responderam que foram até a Unidade para marcar a primeira consulta.

ACS $1-[. .$.$] De rotina, o paciente vem até a$ unidade.

ACS 2-[...] Com o antigo dentista, eram de dois a três por agente de saúde, com o novo dentista, ele manda trazer e avalia o caso, está bem melhor [...]
ACS 3 - [...] São marcados quinta-feira pela manhã na Unidade [...] a CD marca de acordo com a agenda dela.

O procedimento para a marcação da primeira consulta odontológica mostrou-se variável entre as USFs, sendo possível identificar a predominância dessa marcação pelo atendimento da demanda espontânea e quase ausência da oferta organizada de serviços, defendida pelo modelo de Vigilância da Saúde (TEIXEIRA; PAIM; VILASBÔAS, 1998).

A fala do CD2 revela uma preocupação no sentido de se priorizarem os atendimentos:

CD2 - [...] Todo começo de mês, abrem-se va-
gas e encaminhamos para o andamento do mês.
No primeiro dia, faz triagem e os pacientes já
saem com a data de volta. As prioridades são as
urgências e necessidades do paciente. Essa for-
ma de organizaçáo é protocolo da secretaria de
saúde do Município.

Aparece aqui uma diretriz da gestão no sentido de se fazer uma 'triagem' com base nos achados clínicos para a identificação de prioridades. Mas será que esse procedimento pode ser caracterizado como estratégia para a organização da oferta da primeira consulta, conforme prevê o modelo de Vigilância da Saúde?

A necessidade de se identificarem pessoas ou grupos de risco permite que o serviço de saúde estabeleça critérios para priorizar o atendimento de acordo com o agravo à saúde ou grau de sofrimento. A adoção desse critério deve ser baseada nas necessidades da população a partir de levantamentos epidemiológicos das áreas de abrangência das unidades de saúde de forma equânime e universal, devendo a comunidade participar ativamente por meio dos conselhos de saúde municipais (BRASIL, 2006).

Para Pereira (2002), o conhecimento da existência de maior risco para alguns indivíduos ou segmentos da população é utilizado pelos profissionais de saúde, sendo conhecido como enfoque de risco. Os fatores de risco podem se relacionar às características individuais ou 
ambientais e associar-se diretamente à maior ou menor possibilidade de adoecer.

O conhecimento do território e da população, bem como da dinâmica familiar e social deve fazer parte da rotina de trabalho das Equipes de Saúde da Família e trazem dados importantes para o planejamento, acompanhamento e avaliação das ações. Compóem essa rotina de trabalho o mapeamento da área de abrangência (realização e atualização) com identificação das áreas de risco, a identificação de pessoas e famílias em situação de risco e vulnerabilidade, o cadastramento das famílias e a atualização das informaçôes (BRASIL, 2006).

Diante disso, a 'triagem' que orienta a marcação da primeira consulta odontológica, citada pelo CD2, a partir do exame clínico de usuários que buscam a USF, limita-se a uma análise individual e a partir de uma demanda espontânea, não se constituindo, portanto, numa estratégia que possibilite identificar pessoas e grupos da área de abrangência expostas ao maior risco e vulnerabilidade. $\mathrm{O}$ atendimento à demanda espontânea é importante e necessário, mas não deve se constituir como fio condutor na organização dos serviços de saúde, sob pena de não se avançar na mudança do modelo de atenção, persistindo-se nas práticas com foco na doença.

Para Souza e Roncalli (2007), o cumprimento de princípios do SUS, tais como a universalidade e a equidade, requer das ESBs o conhecimento da realidade local e da comunidade adscrita. Daí a necessidade de se organizarem ações valendo-se de estratégias de programação que utilizem critérios para a priorização da população mais necessitada.

Carnut et al. (2011) propuseram um Índice de Necessidade de Atenção à Saúde Bucal (INASB) que utiliza informaçóes da ficha $A$, podendo contribuir como instrumento de programação para ESBs ao permitir também a aproximação com as condições sociais das famílias.

Ainda sobre a primeira consulta odontológica, outras propostas emergiram:

CD1-[...] Segunda idoso, terça pacientes especiais, diabéticos, hipertensos, quarta as crianças [...] Os agentes comunitários raramente trazem pacientes [...] antigamente os agentes (ACS) podiam trazer cinco pacientes, mas nem todos eram atendidos e gerava muita confusáo [...] $O$ dentista anterior foi quem organizou desse jeito e eu dei continuidade.

Essa resposta revela a iniciativa de organização da oferta da primeira consulta odontológica a partir das linhas do cuidado, unificando-se a porta de entrada com o serviço médico e de enfermagem como recomendado pelas diretrizes da PNSB (BRASIL, 2006). Ou seja, é sinalizado aqui um trabalho mais articulado entre a ESB e a Equipe de Saúde da Família na direção à integralidade da atenção. No entanto, é pertinente lembrar a ressalva apontada na Portaria 2488/2011, no sentido de não se transformarem essas linhas de cuidado em novas barreiras de acesso (BRASIL, 2011). Em outras palavras, há que se ter um equilíbrio entre as atividades para grupos de risco e demandas por assistência individual, pois usuários que não fazem parte desses grupos se sentem menos 'privilegiados', como aponta estudo de Cunha e Vieira-da-Silva (2010).

Essa postura, aparentemente desvinculada de reflexão da prática vigente ou mesmo daquela sugerida pela gestão de saúde bucal, pode ser observada quando o CD1 conclui dizendo que: "[...] O dentista anterior foi quem organizou desse jeito e eu dei continuidade [...]".

Finalizando essa categoria:

CD3 - [...] Marcação toda quinta, mais ou menos 35 pacientes. É a própria unidade que faz essa organização, e minha secretária controla agenda.

Caberia perguntar, primeiramente, de que 'secretária' se trata, visto que essa categoria profissional não consta nem da equipe mínima, nem da equipe ampliada (BRASIL, 2011).

"[...] É a própria unidade que faz essa organização, $e$ minha secretária controla agenda". Esse fragmento indica o distanciamento desse profissional com os propósitos da ESF, indo ao encontro de alguns achados de Chaves e Silva (2007) que constatam a lógica dominante do setor privado sobre o setor público; talvez isso explique sobre 'a secretária' nesse discurso. Estas autoras consideram, ainda, que para o trabalho neste campo, são necessárias 
competências adicionais ao que comumente se denomina 'perfil' para se trabalhar na esfera pública. Este fragmento denuncia a falta de competência no campo das tecnologias das relaçóes para o estabelecimento de vínculo com a comunidade adscrita, dificultando a prática do acolhimento. A ausência do acolhimento interfere na garantia do acesso e na priorização do atendimento (COELHO; BESSA JORGE, 2009).

\section{A PARTICIPAÇÃO DA COMUNIDADE}

Todos os sujeitos da pesquisa foram questionados sobre a existência da participação da comunidade nas decisóes sobre a organização e distribuição das vagas para tratamento odontológico. Obtiveram-se como respostas as seguintes falas:

US (todos) - Não sei.

ACS1 - [...] Houve várias reuniōes decidindo que a melhor forma de organizar é o usuário vir até o posto. Não houve participação da comunidade.

ACS2 - A comunidade nunca participou. Agora tem reunióes com os agentes comunitários $e$ a parte odontológica. Os ACS representam os usuários.

ACS3 - Eu nunca participei, mas creio que já tenha tido [...]

CD2 - São os dentistas e ACS que organizam e distribuem.

As falas acima permitem inferir que a comunidade não tem participado nas decisóes tomadas nas USFs no que se refere à organização da oferta dos serviços de saúde bucal na ESF.

Chama à atenção o fato de o ACS2 mencionar que os usuários são 'representados' pelos Agentes Comunitários de Saúde, desconsiderando o aporte legal que sustenta a participação social no SUS, com a definição dos segmentos de trabalhadores, usuários, prestadores e gestores.
A participação popular ou o controle social, um dos princípios norteadores do SUS, se ampara na Lei Federal 8.142/90, que instituiu os Conselhos e Conferências de Saúde, e, posteriormente, na Resolução 333 de 2003, do Conselho Nacional de Saúde (BRASIL, 2003). Atualmente, as instâncias de participação podem se capilarizar até o nível dos Conselhos Locais de Saúde, sendo possível a existência de Unidades de Saúde, com seu respectivo Conselho, como um espaço de participação da comunidade que realmente utiliza o serviço.

No entanto, neste mesmo País das grandes desigualdades sociais, a participação popular, não por acaso, é muitas vezes fraca e desarticulada. Bastos et al. (2009) relembram que a formação do Estado brasileiro precedeu à formação da própria sociedade como entidade anterior forte e robusta, tendo distribuído recursos e serviços de maneira desigual, bem como espaços de poder privilegiado aos grupos dominantes. Essa configuração estatal, resultante de um modelo de colonização de exploração, opera de modo relativamente independente de uma sociedade fragmentada.

Nesse sentido, Roncalli (2000) ressalta que a introdução do controle social foi vista com muito ceticismo por uma boa parte dos profissionais de saúde coletiva, pois consideravam a população brasileira despreparada em termos de consciência política, permitindo práticas clientelistas.

De fato, os trabalhadores do SUS entrevistados neste estudo parecem 'não acreditar' no Controle Social, tomando as decisóes apenas entre os profissionais envolvidos e deixando de fora a comunidade, como explícito na fala do CD2.

Diante disso, cabe perguntar em que cenário se pode praticar a promoção da saúde.

A carta de Ottawa defende a promoção da saúde como empoderamento da comunidade, tanto para identificar como para enfrentar as condiçóes associadas ao processo saúde-doença (WESTPHAL, 2006).

O empoderamento da comunidade também deve ser objeto de trabalho da ESF, indo além do desenvolvimento das habilidades individuais para o autocuidado - como proposto num dos campos de ação da Promoçáo de Saúde -, avançando para o empoderamento político no sentido do fortalecimento da 'consciência política', 
essencial para a construção das Políticas Públicas Saudáveis, rumo à superação dos contextos desfavoráveis à vida.

A participação dos usuários nas decisóes tomadas pelo serviço de saúde não se refere 'apenas' à luta pelo direito individual à assistência. Muito mais do que isso, tem o potencial de traçar os horizontes sobre a própria consolidação do Sistema Único de Saúde no Brasil.

\section{ACESSIBILIDADE AO SERVIÇO DE SAÚDE BUCAL}

No campo da avaliação dos serviços de saúde, a acessibilidade pode ser definida como as características do serviço que permitem mais fácil utilização pelos usuários (UNGLERT, 1995). Seu autor propóe que a acessibilidade seja considerada em três dimensóes: acesso geográfico, acesso econômico e acesso funcional. Para este estudo, chamaram atenção as interferências negativas - as barreiras - sobre o acesso funcional. Ou seja, aqueles aspectos de funcionamento dos serviços de saúde que interferem em sua plena utilização.

Ao serem questionados sobre os problemas e dificuldades em relação ao acesso à primeira consulta odontológica, emergiram as seguintes respostas:

US1-[...] às vezes o aparelho está quebrado e as pessoas que estavam marcadas vão ficando para outro dia.

ACS2 - [...] Equipamentos quebrados e falta de material.

CD1 - [...] Quando o compressor quebra, ai para tudo [...] o paciente que está em tratamento atrasa, e o que está na fila para primeira consulta precisa esperar mais [...] precisava mudar algumas coisas; tem material para trabalhar, mas faltam instrumentais.

Percebe-se nas falas acima que a falta de materiais, instrumental e manutenção de equipamentos constituem-se barreiras organizacionais, aumentando o tempo de espera para o atendimento e agravando a demanda reprimida existente:
ACS 1 - [...] mas a demanda é muita para pouca vaga.

US 2 - [...] muita gente procura tratamento odontológico para poucas vagas.

US 3 - [...] muitas pessoas procurando pelo atendimento, ai nem sempre consegue atendimento.

A situação retratada é coerente com os números sobre a cobertura de serviços de saúde bucal na ESF desse Município, que alcança apenas $20,3 \%$ da população (BRASIL, 2012).

Diante do exposto, além da ampliação da cobertura, recomenda-se que o Município adotado como campo deste estudo utilize o arsenal metodológico do levantamento epidemiológico para a identificação dos grupos e indivíduos vulneráveis ao risco e discuta os achados junto à comunidade, fomentado a participação social, inclusive no que tange à melhoria do acesso ao serviço de saúde, e permitindo, assim, o empoderamento desse grupo e a adoção da universalidade e equidade como princípio fundamental à organização do acesso aos serviços de saúde bucal.

\section{Considerações finais}

Sobre a organização da oferta dos serviços de saúde bucal na ESF no Município estudado, foi possível verificar a existência de heterogeneidade quanto às condutas das ESBs, predominando a triagem dos usuários que buscam pelo serviço, numa maior aproximação do atendimento tradicional da demanda espontânea.

Não foi possível perceber a participação da comunidade na organização da oferta dos serviços prestados pelas ESBs, revelando a fragilidade do Controle Social.

Quanto à acessibilidade aos serviços de saúde bucal na ESF, foi possível detectar barreiras funcionais pela falta de materiais e de manutenção de equipamentos, comprometendo a plena utilização da capacidade instalada dos serviços existentes. 


\section{Referências}

ANTUNES, J.L.F.; NARVAI, P.C. Políticas de saúde bucal no Brasil e seu impacto sobre as desigualdades em saúde. Revista de Saúde Pública, v.44, n.2, p.360-365, 2010.

BARDIN, L. Análise de conteúdo. Lisboa: Edições 70, 1979.

BASTOS, F.A.; SANTOS, E.; TOVO, M.F. Capital social e Sistema Único de Saúde (SUS) no Brasil. Saúde e Sociedade, v.18, n.2, p.177-188, 2009.

BRASIL. Ministério da Saúde. Portaria no 333, de 04 de novembro de 2003. Diretrizes para criação, reformulação, estruturação e funcionamento dos Conselhos de Saúde. Diário Oficial da União, Poder Executivo, Brasília, 4 de dezembro de 2003. Seção 1, p.57.

BRASIL. Ministério da Saúde. Sala de situação em saúde. Ações de saúde. Disponível em: <http://189.28.128.178/sage>. Acesso em: 17 jul. 2012.

BRASIL. Ministério da Saúde. Secretaria de Atenção à Saúde. DATASUS. Cadastro Nacional de Estabelecimentos de Saúde. Disponível em: <http://cnes.datasus.gov.br/ Mod_Ind_Equipes.asp?VEstado=29\&VMun $=291920 \&$ VComp $=201104>$. Acesso em: 14 jun. 2011.

BRASIL. Ministério da Saúde. Secretaria de Atenção à Saúde. Departamento de Atenção Básica. Coordenação de Saúde Bucal. Diretrizes da Política Nacional de Saúde Bucal. Brasil, 2004. Disponível em: <http://bvsms.saude.gov.br/bvs/publicacoes/politica_nacional_brasil_sorridente.pdf>. Acesso em: 30 jan. 2013.

Primeiros resultados do Projeto SBBrasil são anunciados pelo Ministério da Saúde. Disponível em: <http://www.sbbrasil2010. org >. Acesso em: 14 jun. 2011.

BRASIL. Ministério da Saúde. Secretaria de Atenção Básica. Cadernos de atenção básica n 17: Saúde Bucal: Ministério da Saúde, 2006. p.21-24.

BRASIL. Portaria GM/MS n²488, de 21 de outubro de 2011. Aprova Política Nacional de Atenção Básica, estabelecendo a revisão de diretrizes e normas para a organização da Atenção Básica, para a Estratégia de Saúde da Família (ESF) e o Programa de Agentes Comunitários de Saúde (PACS). Diário Oficial da União, Poder Executivo, Brasília, 21 out. 2011.

CARNUT, L. et al. Validação inicial de necessidade de atenção à saúde bucal para as equipes de saúde bucal na estratégia de saúde da família. Ciência e Saúde Coletiva, v.16, n.7, p.3083-3091, 2011.
CHAVES, S.C.L.; DA SILVA, L.M.V. As práticas profissionais no campo público de atenção à saúde bucal: o caso de dois municípios da Bahia. Ciência e Saúde Coletiva, v.12, n.6, p.1697-1710, 2007.

COELHO, M.O.; BESSA JORGE, M.S. Tecnologia das relações como dispositivo do atendimento humanizado na atenção básica à saúde na perspectiva do acesso, do acolhimento e do vínculo. Ciência e Saúde Coletiva, v.14, supl.1, p.1523-1531, 2009.

CUNHA, A.B.O.; VIEIRA-DA-SILVA, L.M. Acessibilidade aos serviços de saúde em um município do estado da Bahia, Brasil, em gestão plena do sistema. Cadernos de Saúde Pública, v.26, n.4, p.725-737, 2010.

DE SOUZA, T.M.S, RONCALLI, A.G. Saúde Bucal no Programa Saúde da Família: Uma Avaliação do modelo assistencial. Cadernos de Saúde Pública, v.23, n.11, p.2727-2739, 2007.

FRAZÃO, P; NARVAI, P.C. Saúde bucal no Sistema Único de Saúde: 20 anos de lutas por uma política pública. Saúde em Debate, v.33, p.64-71, 2009.

INSTITUTO BRASILEIRO DE GEOGRAFIA E ESTATÍSTICA (IBGE). Censo 2010: cidades. Disponível em: <http://www.ibge.gov.br/cidadesat/topwindow.htm?1>. Acesso em: 17 jul. 2012.

MINAYO, M. C. S. O desafio do conhecimento: pesquisa qualitativa em saúde. 11. ed. São Paulo: Hucitec, 2008.

PEREIRA, M.G. Serviços de Saúde. In: PEREIRA, M.G. Epidemiologia: teoria e prática. 6. ed. São Paulo: Guanabara Koogan, 2002. cap. 23, p.513-537.

PROGRAMA DAS NAÇÕES UNIDAS PARA DESENVOLVIMENTO (PNUD). Relatório de desenvolvimento humano 2010. Disponível em: <http://hdr.undp.org/en/reports/global/hdr2010/chapters/ pt/>. Acesso em: 14 jun. 2011.

RONCALLI, A.G. Organização da Demanda em Serviços Públicos de Saúde Bucal: universalidade equidade e integralidade em saúde bucal. 2000. Tese (Doutorado em Odontologia Preventiva e Social) - Faculdade de Odontologia de Araçatuba, Universidade Estadual Paulista Júlio de Mesquita, Araçatuba, 2000.

TEXEIRA, C.F.; PAIM, J.S.; VILASBÔAS, A.L. SUS, modelos assistenciais e vigilância da saúde. Informe Epidemiológico do SUS, v.8, n.2, p.728, 1998.

UNGLERT, C.V.S. Territorialização em sistemas de saúde. In: 
MENDES, E.V. (Org.). Distrito Sanitário. São Paulo: Editora Hucitec. Rio de Janeiro: ABRASCO, 1995. p.221-235.

WESTPHAL, M. F. Promoção da saúde e prevenção de doenças. In: CAMPOS et al. Tratado de Saúde Coletiva. São Paulo: HUCITEC, 2006. cap.19, p.635-668.

Recebido para publicação em agosto de 2013

Versão final em dezembro de 2013

Conflito de interesse: não houve

suporte financeiro: inexistente 